\title{
Percurso metodológico para análise de sites na internet
}

\author{
Methodological course for analysis of websites
}

\author{
Rejane Ramos Machado \\ Doutora em Ciências PPGICS/ICICT/FIOCRUZ \\ Laboratório de Informação Científica e Tecnológica em Saúde/Instituto de Comunicação e Informação \\ Tecnológica em Saúde/FIOCRUZ \\ Lidiane dos Santos Carvalho \\ Doutora em Ciência da Informação UFRJ \\ Laboratório de Informação Científica e Tecnológica em Saúde/Instituto de Comunicação e Informação \\ Tecnológica em Saúde/FIOCRUZ
}

\begin{abstract}
Resumo: Esta pesquisa objetiva apresentar critérios metodológicos para análise de sites a partir do espaço atribuído ao campo da Ciência da Informação (CI) e como ela pode contribuir para os estudos de recuperação da informação por parte do usuário. Como método para a construção destes critérios, empregase revisão de literatura em base de dados especializadas e observação empírica com participação do especialista. Como resultado, observa-se que a organização da informação não é estática e está em constante processo de revisão e ajustes. Os eixos utilizados para identificar informações nos sites pelo especialista foram: conteúdo, credibilidade, design e legibilidade. Identificou-se que muita informação sobrecarrega o usuário. O fato de ser sucinta, resumida em sua proporcionalidade são quesitos para melhorar a capacidade de entendimento de quem busca e filtra a informação. Considera-se finalmente que estudos de usuários e sistemas de representação da informação, sejam incluídos na etapa anterior a estratégia de implementação de sites, quanto a sua utilidade e aplicabilidade.
\end{abstract}

Palavras-chave: Análise de sites. Fontes de informação. Informação e saúde.

\begin{abstract}
This research presents methodological criteria for site analysis from the space assigned to the field of Information Science (CI) and how it can contribute to the studies of information retrieval by the user. As a method for the construction of these criteria, includ the literature review used in a specialized database and empirical observation with expert participation. As a result, the presents of the view the organization of the information is not a static and constant process of revision and adjustments. The axes used to identify information on the sites by the specialist were: content, credibility, design, and readability. It has detected that a lot of information overloads the user. The fact of being brief, summarized in its proportionality are questions to improve the capacity of understanding of those who search and filter the information. Finally, considered the significative importance the "user studies" and information representation systems for the make a stage of the strategy of site implementation, as well as their usefulness and applicability.
\end{abstract}

Keywords: Site analysis; Information sources; Health information. 


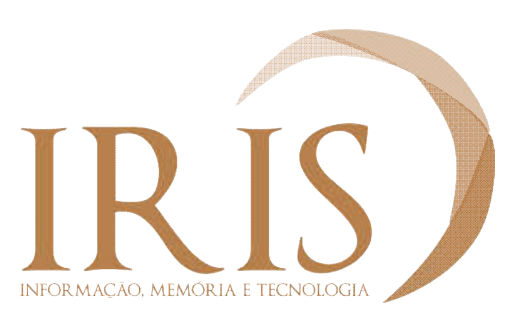

Percurso metodológico para análise de sites na internet Rejane Ramos Machado e Lidiane dos Santos Carvalho

\section{Introdução}

No campo dos estudos sobre emergências em saúde no Brasil, existe uma lacuna no esforço do Poder Público em relação às doenças infecto-parasitárias visando o seu controle e combate e persistem ainda as doenças tais como, leishmaniose, leptospirose, entre outras, que colocam em risco a saúde da população. Para resolver parte destas emergências de saúde, o governo brasileiro tem reunido esforços na produção, promoção e disponibilização de conteúdos informativos em sites do governo na Internet, trazendo informações sobre as formas de prevenção de doenças infectoparasitárias com vistas a promoção da saúde no País.

Entretanto, cabe destacar que a percepção do usuário sobre determinado conteúdo informativo, requer sobretudo reconhecer a dimensão cognitiva deste processo, pois, envolve modificar os limites e o entendimento dos conceitos embutidos nesses conteúdos, de modo que esteja inserido em uma lógica ou percepção ou imaginação, que seja de domínio do usuário que busca a informação.

No que diz respeito à saúde é fundamental, conhecer a informação que está indexada, qualificada e organizada em fontes de informação sobre as doenças, incluindo a prevenção e redução de riscos de modo que possa ser acessada pelos cidadãos de um modo geral. Essas informações contêm elementos norteadores sobre as formas de prevenção e controle dessas doenças pela população.

Este artigo situa e recapitula o espaço atribuído ao campo da Ciência da Informação (CI) e como ela pode contribuir para a os estudos de recuperação da informação por parte do usuário. Para além disso, no que diz respeito a representação da informação constitui-se em sua dimensão prática, mapear e analisar as bases de dados do poder público sobre as doenças infecto-parasitárias, visando conhecer o propósito de sua organização orientada para o usuário, faz-se necessário o entendimento da 'representação da informação' e sua aplicabilidade, considerada como área fundamental da Biblioteconomia que está envolvida com os processos de organização da informação (na classificação por assunto), dos estoques informacionais e na construção de modelos e formas de descrição de conteúdo. Como espaço, o atuar no campo informacional para a promoção da saúde, envolve conhecer como o cidadão se apropria e compreende a informação sobre as doenças de modo que o mesmo possa apreendê-la. 
Entende-se que é um desafio para a pesquisa científica mapear o grupo populacional que está mais exposto ao risco de contrair essas doenças. Além desse mapeamento, há o desafio de se chegar a esse grupo e conseguir que os mesmos tenham acesso à informação de suas condições socioambientais, bem como criar estratégias para dirimir essa vulnerabilidade. Neste artigo, são apresentados os resultados da análise das informações disponíveis nos sites do governo. A relevância desse trabalho está associada a apresentação de um arcabouço teórico metodológico que pode contribuir sistematicamente para avaliação e seleção de fontes de informação na internet, construção de políticas de representação da informação.

Os preceitos teóricos que orientam este trabalho passam por três conceitos, são eles ciberespaço, tecnologia e representação da informação. O primeiro conceito trata da caracterização do espaço social que conhecemos como internet. Para este momento, apropria-se do conceito de ciberespaço como aquele aonde a virtualidade reúne formas materializadas da ação humana. Sabe-se que a internet na sua essência possibilita a comunicação entre muitos de forma atemporal e numa expansão global. Para Lévy (2010, p. 94-95) o ciberespaço “tem a vocação de colocar em sinergia e interfacear todos os dispositivos de criação de informação, de gravação, de comunicação e de simulação" sendo "um dos principais motores da cibercultura".

O segundo conceito é o de tecnologia que é considerado um determinado conhecimento e sua aplicação no uso de um ferramental desde o mais simples, e aí se inclui os processos mais antigos: como converter ou transformar, para que seja mais adequado à necessidade de um determinado momento. Isso, pode ser um dispositivo, um processo, uma máquina, uma técnica entre outras. Atualmente, existem diversas tipologias para a tecnologia, tais como: tecnologia da educação, da informação, militar, medicinal e social. Para esse trabalho vamos considerar a tecnologia como um "processo envolvendo humanos e não somente artefatos. Para Hughes tecnologia é "como um meio para moldar um cenário" (HUGHES, 2004, p.3).

No ambiente onde reside o volume crescente de produção de informação e consequente circulação de forma pulverizada, a questão é como acumular e de que forma organizar informações afins, armazenadas, categorizadas e ou codificadas em bases de dados que estão acessíveis. Além disso, como pode modificar os limites e o entendimento dos conceitos, embutidos nesses conteúdos, para que esteja inserido em uma lógica ou percepção ou imaginação, que seja de domínio do usuário que busca a informação. Entendendo que a tecnologia e a informação são fenômenos humanos e como tal se constituem em pré-requisitos para fazer valer o direito à informação. 
Consoante emerge à necessidade de se conhecer a informação que está indexada, qualificada e organizada em fontes de informação sobre as doenças incluindo a prevenção e redução de riscos, para ser acessada pelos cidadãos de um modo geral. Essas informações contêm formas de prevenção e controle dessas doenças pela população.

Para essa contextualização, precisamos recapitular o espaço atribuído ao campo da Ciência da Informação (CI), sua evolução e limites. Esse campo surgiu após a Segunda Guerra Mundial, juntamente com outros novos campos. Alguns atores são considerados nesse processo de construção do campo, podemos citar Vannevar Bush, as instituições, e eventos que foram chaves para nortear o caminhar nessa complexa construção do campo. Bush identificou "o problema da explosão informacional - o irreprimível crescimento exponencial da informação e de seus registros, particularmente em ciência e tecnologia” (SARACEVIC, 1996, p.42).

Com a revolução científica ocorreu o aumento das publicações tanto científicas quanto técnicas, o registro de todo o tipo de informação, aliado à evolução dos métodos para o processamento da informação e com o surgimento da World Wide Web em meados nos anos 1990. Emergiram mudanças e novos estudos sobre o campo informacional da CI. Um desses é a 'relevância' como atributo que reflete a eficácia na troca de informação entre usuários e sistemas de recuperação de informação, em contextos e aplicações mais específicas. Saracevic (1996), indica tipos de relevância: algorítmica, assunto, cognitiva, situacional e motivacional. Essa tipologia era utilizada não para avaliar somente o sistema, mas, visando a relevância cognitiva e sua utilidade, para um determinado usuário, para que o sistema fosse aceitável. A dinâmica do processo cognitivo é compreendida via estrutura mental e suas manifestações. Nesse aspecto a CI, em seus estudos incorpora as questões da cognição humana, tendo essa como o consumidor de informação. Portanto, busca olhar de forma mais prospectiva a estrutura da representação. Passa então, a se constituir um deslocamento do processamento da informação que incluía a descrição e a representação, como parte da RI.

A representação da informação passa a ser a área central do campo e como objeto de pesquisa da CI está voltada para discussões teóricas sobre o campo representacional e sobre o desenvolvimento de sistemas de informação, mas sempre relacionada "da possibilidade de melhorar os processos representacionais, construindo linguagens melhores, [...] classes mais consistentes, terminologias menos ambíguas" (ARAÚJO, 2009, p.201). As linguagens representacionais surgiram no esforço para a recuperação de perdas nos métodos de indexação por assuntos e especificidades. 
O autor menciona também, que na busca por uma completeza (o estado de completo) na construção da 'linguagem perfeita' não foi colocado como relevante o sujeito que busca essa informação. É razoável conhecer as características da CI que são as três ideias que fundamentam esse campo: processamento, a relevância e a interação.

Esse breve panorama teórico aqui traçado diz respeito aos fatos ocorridos nas últimas décadas, aonde situa-se a internet, a tecnologia, a informação e a representação da informação. Estes fenômenos socio-técnicos, têm papel relevante para a organização da informação, no contexto das novas tecnologias de informação e comunicação que vem se desenvolvendo de forma acelerada, compondo o cenário do cotidiano dos usuários. Portanto, se faz necessário o refinamento da informação que possa satisfazer as reais necessidades dos usuários, informações essas que são complexas e diversificadas. Essa visão vem para possibilitar a compreensão de que forma as escolhas acontecem.

\section{Método}

Os procedimentos metodológicos consistem em três etapas. A primeira de revisão de literatura consiste na identificação, leitura e sistematização de literatura referente a representação da informação. E a segunda etapa da sistematização desta literatura confecciona o conteúdo para construção de eixo estruturante de avaliação de site e a terceira propriamente dita é a própria avaliação de sites. A segunda e terceira etapa são pertencentes aos resultados.

Para a primeira etapa foi feita identificação, a partir de vocabulário estruturado trilíngue DeCS - Descritores em Ciências da Saúde, desenvolvido pela Bireme (decs.bscalud.org), a partir do Medical Subject Headings (MeSH) da National Library of Medicine (NLM). Essa estrutura tecnológica contém terminologia única, agregando conceitos que são organizados em uma estrutura hierárquica. Essa estrutura com vocabulário dinâmico, com revisão e atualização periódica, possibilita a formação de buscas temáticas, que é um elemento facilitador para a construção de estratégia, para recuperação da literatura do conjunto de temas aqui abordados.

Com a terminologia identificada nos variados aspectos do projeto, passa-se para a etapa de levantamento bibliográfico, construindo estratégia de busca nas principais bases de dados. 
Para essa etapa a busca pela literatura pertinente foi realizada nas bases Scopus e Web of Science com os seguintes termos: ("science information theory") AND SUBJAREA (mult OR arts OR busi OR deci OR econ OR psyc OR soci) AND PUBYEAR > 1999 AND (LIMIT-TO (SUBJAREA, "COMP") OR LIMIT-TO (SUBJAREA, "DECI") OR LIMIT-TO (SUBJAREA, "SOCI")) AND (LIMIT-TO (LANGUAGE, "English") OR LIMIT-TO (LANGUAGE, "Portuguese") OR LIMITTO (LANGUAGE, "Spanish")). Cabe esclarecer que essa estratégia foi considerada ponto de partida para o trabalho e mantida na atualização da busca bibliográfica. Os registros recuperados foram migrados para o gerenciador de referências Mendeley, para a retirada das duplicatas e categorização dos resultados. Além disso o procedimento de revisão da literatura nas bases de dados, foi abrangente o suficiente sobre a representação da informação.

Sobre as buscas na base a respeito das doenças infecto parasitárias foi necessário identificar quais palavra-chave indexam as publicações científicas sobre essas doenças. Retorna-se então ao DeCS, no índice hierárquico com navegação nas categorias: classificação de doenças $(C)$ e saúde pública (SP). O uso do índice hierárquico possibilita identificar em que ponto da estrutura os termos estão inseridos. Identifica-se que inexiste no DeCS o descritor doença infecto-parasitária, sendo necessária a busca do termo de forma separada. Identifica-se também que alguns termos constam como sinônimos: doenças contagiosas, doenças infecciosas e enfermidades transmissíveis. Os termos identificados na estrutura hierárquica do DeCS, nos três idiomas com as respectivas definições de cada termo extraído são considerados representativos das doenças que de alguma forma estão presentes no ambiente.

Algumas categorias de doenças pesquisadas para coleta dos dados, foram aventadas, doenças oriundas de saneamento inadequado e que estão categorizadas como parasitárias como, por exemplo, a teníase, filariose, helmintíase, esquistossomose e leishmaniose. Essas doenças são transmitidas seja por insetos, contato com água, orofecal e as relacionadas à higiene.

O poder público tem como premissa a preocupação explicitada na Resolução n ${ }^{\circ}$, de 29 de julho de 2002 ${ }^{1}$, onde em seu Capítulo I no artigo $3^{\circ}$ define que a informação em um site governamental apresentada de forma clara seu propósito, a sua abrangência e definição do público alvo, além da verificação de outros sites com o mesmo propósito. Considerando essa premissa, critérios devem ser estabelecidos para a análise e avaliação das informações prestadas por estes sites instituídos pelo governo. A partir de literatura sobre essa temática, foram identificados e relacionados os sites a serem analisados. Os sites identificados estão mencionados no Quadro 1. Os 


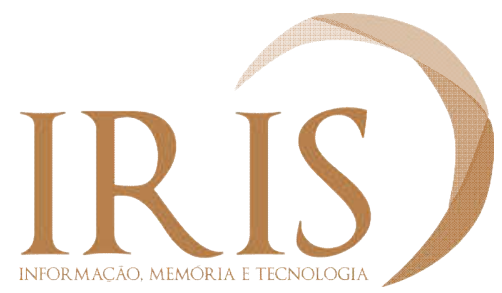

Percurso metodológico para análise de sites na internet Rejane Ramos Machado e Lidiane dos Santos Carvalho

procedimentos de busca e coleta de dados foi realizada e logo em seguida a análise foi feita pelo especialista. Utilizou-se a ferramenta de busca do google, em português, considerando que seria um comportamento inicial do usuário reportar-se a este buscador, para identificar se os termos levariam a algum achado sobre as doenças nesses sites.

Para a coleta de dados foi elaborado instrumento inicialmente em arquivo Excel para facilitar a coleta e retorno dos dados e a migração para software de análise qualitativa (MAXQDA). Nesse instrumento contém os pontos a serem observados e descritos durante a análise de cada unidade. A análise foi realizada individualmente por três especialistas, dois da área de biblioteconomia e um da área de tecnologia. Cabe acrescentar que nessa coleta busca manter o comportamento de um usuário, com cabedal de conhecimento tácito. Os dados coletados são transcritos para análise na etapa dos resultados.

As unidades de análise dispostas por critérios atribuídos no teor dos atributos dispostos dessa forma: conteúdo, credibilidade, design e legibilidade. Para a análise dos dados por especialistas, os mesmos são migrados para a estrutura criada no MAXQDA. Essa estrutura no software possui dois sistemas: o de documentos e o de códigos. O sistema de documentos está dividido em três grupos denominados (ESP1, ESP2, ESP3), em cada grupo constam as unidades analisadas, totalizando cinco unidades. O sistema de códigos atribuído está dividido nos atributos e critérios que compõem o checklist delineado para a análise dos sites. A organização da estrutura básica do MAXQDA em janelas, possibilita rearranjos na visualização em forma de colunas sendo útil para o trabalho. O sistema de códigos é a principal ferramenta de análise. Os códigos atribuídos são os atributos considerados no checklist (conteúdo, credibilidade, design e legibilidade).

\section{Resultados}

A segunda etapa que se constitui na análise dos sites utiliza-se como elementos norteadores as diretrizes compiladas a partir de trabalhos considerados com conteúdo para construção de eixo estruturante de avaliação de sites: a) organização Health on the Net Foundation registra os "princípios básicos da ética" na forma de apresentação da informação. Esses princípios estão dispostos no código de conduta (HONCode); b) os critérios de avaliação da qualidade da informação em saúde da American Medical Association publicado no JAMA; c) critérios do Institute for Healthcare Information 
Technology (IHIT) que tem por objetivo a melhoria da vida através de cuidados de saúde; d) Silberg (1997) em trabalho de revisão registra critérios iniciais para avaliação, controle e garantia de qualidade dos sites contendo informação em saúde; e) Eysenbach (2002) que apresenta critérios de qualidade relacionados a Website em saúde (técnico, acurácia, abrangência, inteligibilidade e design). Os trabalhos acima estão entre os mais citados sobre essa temática.

$\mathrm{Na}$ questão ética exemplo é seguido no Brasil pelo CREMESP ${ }^{2}$ que publica esses princípios para nortear uma "política de auto-regulamentação e critérios de conduta dos sites de saúde e medicina na internet”. Estabelecendo uma relação com os cinco critérios de avaliação das fontes impressas, critérios esses já bem conhecidos pela Ciência da Informação, que são acuidade, autoridade, objetividade, atualização e cobertura Mostafa (1998); Lopes (2004). Critérios genéricos são mencionados por Tillman (1997), para identificar a qualidade de recursos da internet: autoridade de autor ou criador; comparabilidade com fontes relacionadas; estabilidade de informações; adequação do formato; requisitos de software, hardware ou multimídia.

Kahn em 1997 delineia o modelo Qualidade da informação / desempenho de produtos e serviços (IQ/PSP) a partir de estudo de caso. Esse modelo é testado e publicado em 2002. Kahn (2002) define a qualidade da informação em quatro dimensões: relevância, interpretabilidade, credibilidade e reputação. Na Tabela 5 é apresentada todas as dimensões estabelecidas no modelo de Kahn. Esses modelos agregados ao de Lancaster (1989) se aproximam dos critérios estabelecidos pela Health Information Technology Institute (HITI) mencionado por Lopes (2004).

Outros trabalhos, tais como, Pereira Neto e Paolucci (2014), apresentam indicadores construídos para avaliar site específico na temática 'dengue', relacionando critérios identificados a partir de Eysenbach e agrupados a saber: técnico, design, legibilidade, acurácia e cobertura. Outra abordagem é feita posteriormente por Mendonça e Pereira Neto (2015), com uma análise da literatura sobre instrumentos de avaliação apresentando uma relação de critérios identificados no teor de cada instrumento.

No texto acima um conjunto de critérios são agrupados em três dimensões: conteúdo, técnica e design. Na continuidade, Pereira Neto (2015) menciona os resultados do processo de avaliação de sites realizados pelo Laboratório Internet Saúde e Sociedade (LaISS), nas temáticas 'dengue, tuberculose e aleitamento materno'. Os critérios considerados foram: abrangência, interatividade, legibilidade, técnico e acurácia. O autor menciona que são aproveitados critérios reconhecidos e consagrados internacionalmente. Os critérios estabelecidos pelo LaISS estão 
disponíveis no site (http://andromeda.ensp.fiocruz.br/). Mesmo utilizando de linguagem ou ponto de vista diferentes, os autores acima mencionados nomeiam e categorizam de forma específica, mas, observa-se que existe uma concordância de ideias no que diz respeito ao método ou metodologia para avaliar as fontes de informação.

Os critérios clássicos de avaliação desenvolvidos e aplicados na biblioteconomia para desenvolvimento de coleções e aquisições de estoques informacionais, foram considerados como eixo básico, entendendo que no meio impresso o objeto (livros, periódicos, etc.) são fontes de informação que diferem dos limites tênues do meio eletrônico. Para se fazer uma atualização ou customização ao meio eletrônico, deve-se considerar que esse meio a princípio não tem critérios de organização, e, o que está inscrito são informações textuais ou imagens. Entendendo um pouco mais sobre critérios, dimensões ou outras nomeações feitas pelos autores acima, a partir de uma visão social ou funcional, Lopes (2008), em sua pesquisa, identifica instituições internacionais que desenvolveram checklist's para avaliar páginas na Web. Hjørland (2011, 2012), trabalha com o modelo denominado 'checklist approach'. Esse modelo tem uma função funcionalista ou instrumental, ou seja, a princípio é detectar se uma fonte é boa ou não. Sabendo que não é só isso que conta, existem outros fatores tais como o quanto essa fonte é citada, a especialidade etc., mas, é uma etapa necessária essa maneira mecânica de iniciar um processo de avaliação. 


\section{IRIS}

Percurso metodológico para análise de sites na internet Rejane Ramos Machado e Lidiane dos Santos Carvalho

Tabela 1 - Lista de verificação para avaliar recursos Web

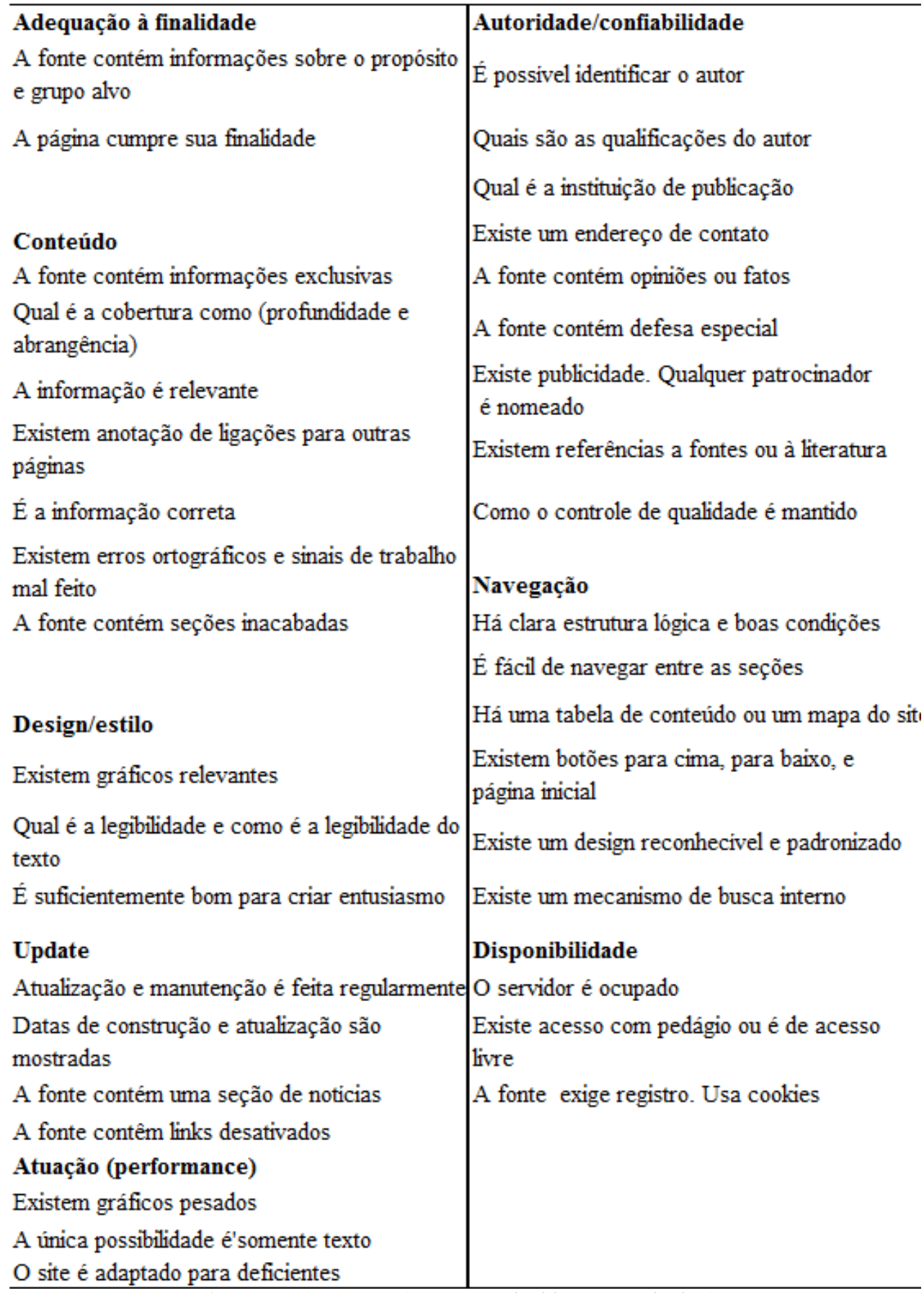

Fonte: HJØRLAND (2011, 2012). Nota: traduzido por Machado (2017, p. 76).

A terceira etapa com o uso da lista de verificação para avaliar recursos Web, o especialista analisa a fonte de informação na web, a partir de pontos listados que são considerados relevantes, 


\section{IRIS}

Percurso metodológico para análise de sites na internet Rejane Ramos Machado e Lidiane dos Santos Carvalho

tais como autoridade, exatidão objetividade, moeda e cobertura do documento mencionado por Hjørland (2011, p.1893; 2012, p.259). Nesse novo momento se insere os critérios aplicados para as fontes de informação impressa, acrescendo atualizações ou conformações para essa nova realidade, considerando a literatura mapeada. Lopes (2004), estabelece uma análise dos critérios disponibilizados por organismos internacionais em suas iniciativas e também as ações no Brasil sobre a avaliação da qualidade da informação em saúde.

Consoante com esse entendimento, a proposta é estabelecer um conjunto de critérios que evolva os princípios da Ciência da Informação no ambiente virtual. Os critérios estabelecidos a partir do que foi identificado na literatura e com resultados reconhecidos e mencionados pelos autores acima, são considerados para essa análise. Os critérios dizem respeito aos atributos: conteúdo, legibilidade, credibilidade e design que irão compor o checklist para a análise dos sites. O checklist é construído com base na prática da autora com o serviço de aquisição de biblioteca e da literatura. Entendendo que são utilizados para a análise dos sites, na visão do especialista, com critérios estabelecidos para análise, no entendimento do que significa cada um deles. Esse entendimento está descrito por autores aqui mencionados. Outro ponto a considerar é que o especialista tem como foco buscar se colocar a partir do olhar de um usuário em busca de informação.

Figura 1 - Critérios e atributos

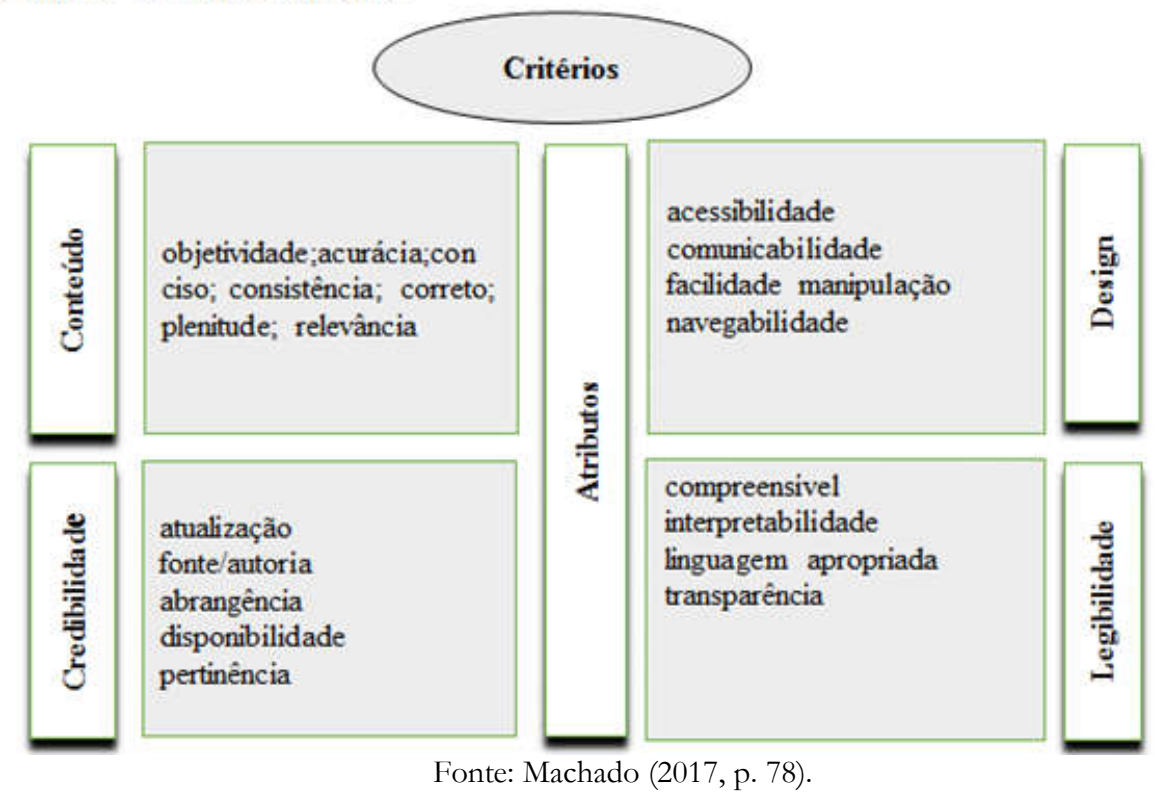


Os sites possuem endereçamento próprio que serve para localizar e identificar computadores. No Brasil, o registro de domínios é feito pelo Núcleo de Informação e Coordenação do Ponto BR (http://www.nic.br/), que é a entidade que implementa as decisões do Comitê Gestor da Internet no Brasil (http://www.cgi.br/). Consoante, existe uma lista de domínios da internet que são atribuídas ao primeiro nível do endereçamento das entidades (TLDs). O endereçamento é completado com a sigla 'url', que representa o localizador padrão de recursos: o endereço virtual. Esse endereçamento pode ser de um site, arquivo, pasta, entre outros. O 'url' é padronizado pela Norma RFC 1738³. Os domínios governamentais são feitos no Núcleo de Informação e Coordenação do Ponto BR (NIC.br), a partir de uma autorização da Secretaria de Tecnologia da Informação (STI). O Comitê Gestor da Internet no Brasil (CGI.br) apregoa o estabelecido na Resolução $\mathrm{n}^{\circ} 8$ de 2008, que traça as diretrizes para registro de domínios das entidades governamentais. Essa resolução é regulamentada pela Portaria no 51, de 7 de outubro de 2016, que regulamenta o processo de autorização de registro de domínios 'gov.br'. Na gestão de domínio, o eGOV analisa as solicitações de registros de domínios das entidades governamentais e sugere qual seria a nomenclatura mais adequada.

Seguindo os critérios adotados para endereçamento e disponibilização de informações em um site, optou-se por utilizar a lista de categorias de domínios já estabelecida e disponível (http://registro.br). Essas categorias de domínio são empregadas no meio virtual para designar qualquer tipo de entidade. Para a seleção foi considerado os resultados visíveis na primeira página, por cada termo pesquisado e a quantidade de resultados recuperados.

São considerados e inseridos nas categorias a saber: na categoria 'com' as empresas e atividades comerciais; em 'edu' as entidades de ensino superior e atividades de educação; 'gov' estão os órgãos do governo (federal, estadual e municipal); em 'inf' estão as bases de dados, bibliotecas digitais, virtuais, repositórios e dicionários e textos científicos; em 'med' sites privados, usualmente na área médica; 'net' as redes e empresas; e em 'org' estão as organizações não governamentais incluindo a Wikipédia, que é assim identificada. Na categoria denominada 'outros' são aglutinados os formatos de imagem e outras mídias. Nessa categorização inicial é considerada a tipologia descrita no endereçamento, em alguns casos é necessário fazer a navegação, já que tem endereçamento não muito claro de sua finalidade. A categoria 'gov' é a considerada pertinente para essa pesquisa, por entender que aglutina informações sobre doenças e o objeto da pesquisa. Não desconsiderando a categoria 'inf' na qual é localizada apresentação em PDF, texto científico, dicionário, biblioteca 


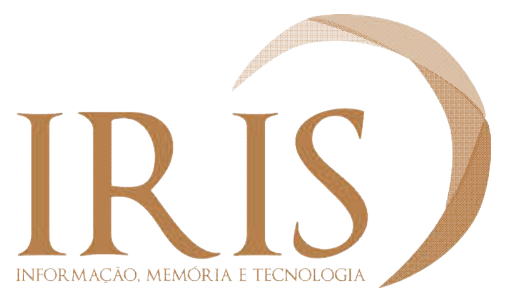

Percurso metodológico para análise de sites na internet Rejane Ramos Machado e Lidiane dos Santos Carvalho

virtual e digital, repositório e informações sintetizadas sobre doenças. Essa análise é feita nos cinco sites identificados, tendo como base critérios aqui explicitados.

Quadro 1 - Endereçamentos de sites do governo identificados por doença

\begin{tabular}{|c|c|}
\hline Doenças & Endereçamentos identificados \\
\hline Esquistossomose & http://portalsaude.saude.gov.br/index.php/oministerio/principal/secretarias/ \\
\hline Filariose & http://portalsaude.saude.gov.br/index.php/oministerio/principal/secretarias/ \\
\hline Leishmaniose & $\begin{array}{l}\text { http } / / \text { portalsaude. saude.gov.br/index.php/oministerio/principal/secretarias/sv } \\
\text { s/leishmaniose-visceral-kv }\end{array}$ \\
\hline Leishmaniose & http:/bvsms.saude.gov.br/bvs/folder/leishmanioses_visceral.pdf \\
\hline Teniase & http://www dive.sc.gov.br/conteudos/zoonoses/publicacoes/Teniase \\
\hline
\end{tabular}

Fonte: Machado (2017, p.81).

A codificação dos dados no software é a parte central de organização do trabalho. Em cada projeto criado, a estrutura é constituída a partir do conhecimento prévio da informação e, de um processo analítico que transcende o fato de se atribuir um código. O passo a seguir é categorizar em códigos e subcódigos e identificar o que é considerado nos achados da análise.

Esses resultados estão dispostos por atributos e critérios. O primeiro atributo diz respeito ao conteúdo que aglutina sete critérios, visando identificar a qualidade da informação que está inscrita, representada textualmente e em outros formatos. O segundo atributo diz respeito a credibilidade que fornece subsídios para acreditação do que está inscrito, o que está registrado, ou representado. O terceiro atributo diz respeito ao design que considera possuir características subjetivas diferentemente do aspecto visual do site, de sua aparência e layout. O quarto atributo diz respeito a legibilidade que é a facilidade de leitura de texto, que está sendo possibilitada por condições favoráveis com uma forma legível, com terminologia adequada para o público leigo.

Os critérios incluídos em cada um desses atributos refletem no seu conjunto as inferências a que estão sujeitos. Em primeiro lugar, cabe o questionamento de como está sendo utilizado o registro de domínio, haja visto que tem maior prioridade o endereçamento por entidade do que por tipo de conteúdo. Em segundo lugar, os sites são incompletos. Em terceiro lugar, em ambiente virtual disponível na internet os erros ortográficos ficam mais visíveis. Em quarto lugar uma informação generalizada torna mais difícil identificar quem é o responsável pelo conteúdo e quais 
critérios científicos basearam a informação representada no site, haja visto que o usuário pode querer saber a respeito da procedência daquela informação. Considerando o estilo da escrita, mais a informação específica, acrescida da questão cultural, esse fato gera resultados promissores de compreensão, interpretação e transparência da informação no atributo legibilidade. Para os especialistas, muita informação sobrecarrega o usuário, o fato de ser sucinta, resumida em sua proporcionalidade são quesitos para melhorar a capacidade de entendimento de quem busca e filtra a informação.

Identifica-se após a análise das unidades acima descritas, que o site do governo mudou sua interface em fins do segundo semestre de 2017. Hoje, está disponível no novo site as secretarias envolvidas e outros órgãos, mas de forma pulverizada. Consta na barra lateral esquerda os assuntos: as informações constantes da Secretaria de Vigilância Sanitária (SVS) estão alocadas no assunto: 'Saúde de A a Z' para diversas doenças. Nos critérios acessibilidade e navegabilidade essa nova disposição continua falha, exigindo um trabalho de navegação para localizar informação sobre a doença, e quanto a acessibilidade a recuperação da página via google não está acessível. Fica claro, que a organização da informação não é estática, estando em constante processo de revisão e ajustes. Os termos são utilizados para identificar informações nos sites pelo especialista.

A análise de sites feita pelos especialistas a plenitude da informação representada, dada como finalizada no contexto da divulgação está aquém dos anseios dos usuários, quanto a sua utilidade e aplicabilidade. $\mathrm{Na}$ continuidade os especialistas consideram que mesmo apresentando a princípio toda a informação de forma categorizada, apenas um site disponibiliza ilustrações, salvo os que são documentos em PDF, não sendo abrangente. Esse critério pertence ao atributo credibilidade.

Pelo apresentado na análise dos sites pelo grupo dos especialistas, considera-se que é ínfima a agregação de elementos originados da realidade tanto cognitiva, quanto das habilidades sociais do usuário. Para os especialistas a informação representada não proporciona uma leitura que gere entusiasmo, pela facilidade de compreensão, e, de estabelecimento de produção de sentido.

\section{Considerações finais}

Este estudo trata de apresentar uma metodologia construída que reúne etapas pragmáticas para a análise de sites. Essa análise no campo da informação em saúde é uma etapa importante para avaliar a qualidade das informações prestadas e disponibilizadas nos sites do governo. A informação para a 


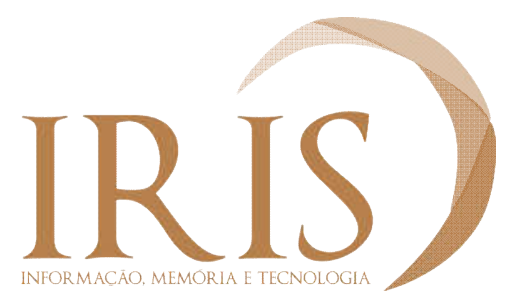

Percurso metodológico para análise de sites na internet Rejane Ramos Machado e Lidiane dos Santos Carvalho

prevenção, promoção e tratamento de doenças deve ser precisa, clara e atualizada. Neste artigo a metodologia proposta contempla estas etapas trabalhando com os atributos conteúdo, credibilidade, design e legibilidade. Apresenta ainda uma lista de verificação para análise dos sites, de acordo com a relevância da informação prestada e sua identificação. Os usuários especialistas apresentam uma maior facilidade no manejo da informação científica em sites governamentais, entretanto, os usuários não especialistas, apresentam uma dificuldade considerável na expressão da sua busca por informação. As observações que compõe a conclusão desta pesquisa, associada a lista da Tabela 1 para avaliar recursos $W e b$ compõe um arcabouço teórico-prático que pode compor recomendação para estudos futuros relativos ao tema e que estes incluam principalmente: a) a replicação deste percurso metodológico como subsídio para o processo de avaliação de sites na internet anterior a propositura de novos conteúdos para os usuários; b) a inclusão nos programas de ensino no campo da saúde deste modelo de análise que possibilite o usuário avaliar criticamente a qualidade da informação ofertada nos sites. Cabe destacar que profissionais do campo da saúde devem estar atentos aos critérios disponibilizados por organismos internacionais em suas iniciativas e também as ações no Brasil sobre a avaliação da qualidade da informação em saúde. Considera-se finalmente que estudos de usuários e sistemas de representação da informação, sejam incluídos na etapa anterior a estratégia de implementação de sites, quanto a sua utilidade e aplicabilidade.

\section{Referências}

ARAUJO, C. A. A. Correntes teóricas da ciência da informação. Ciência da informação, v. 38, n. 3, p. 192204, 2009.

EYSENBACH, G. et al. Empirical studies assessing the quality of health information for consumers on the World Wide Web: a systematic review. Journal American Medical Association, v. 287, n. 20, p. 2691-2700, 2002.

HJØRLAND, B. Methods for evaluating information sources: an annotated catalogue. Journal of Information Science, v. 38, n. 3, p. 258-268, 2012.

HJØRLAND, B. Evaluation of an information source illustrated by a case study: effect of screening for breast cancer. Journal of the American Society for Information Science and Technology, v. 62, n. 10, p. 1892-1898, 2011.

HUGHES, T. P. Human-built world: how to think about technology and culture. Chicago: University of Chicago Press, 2004. 


\section{IRIS)}

Percurso metodológico para análise de sites na internet Rejane Ramos Machado e Lidiane dos Santos Carvalho

KHAN, B. K. et al. Information quality benchmarks: product and service performance. Communications of the ACM, v. 45, n. 4, p. 184-192, 2002.

LÉVY, P. Cibercultura. São Paulo: Ed. 34, 2010.

LOPES, I. L. Novos paradigmas para avaliação da qualidade da informação em saúde recuperada na Web. Ciência da informação, v. 33, n. 1, p. 81-90, 2004.

MENDONÇA, A. P. B.; PEREIRA NETO, A. Critérios de avaliação da qualidade da informação em sites de saúde: uma proposta. RECIIS. Rev. Eletron. de Comum. Inf. Inov. Saúde, v. 9, n. 1, p. 1-15, 2015.

MACHADO, R. R. As características dos sites sobre saúde, ambiente e doenças infecto-parasitárias e o uso das informações pelos jovens circunvizinhos ao Parque Estadual da Pedra Branca na Cidade do Rio de Janeiro. 2017. Tese (Doutorado em Ciências) - Instituto de Comunicação e Informação Científica e Tecnológica em Saúde, Fundação Oswaldo Cruz, Rio de Janeiro, 2017.

MOSTAFA, S. P.; TERRA, M. As fontes eletrônicas da informação: novas formas de comunicação e de produção do conhecimento. São Paulo em Perspectiva, v. 12, n. 4, p. 1-12, 1998.

PEREIRA NETO, A.; PAOLUCCI, R. Qualidade da informação em sites de dengue: análise de uma experiência inovadora. Rio de Janeiro: ENSP/FIOCRUZ, 2014.

SARACEVIC, T. Ciência da informação: origem, evolução e relações. Perspectiva em Ciência da Informação, v. 1, n. 1, p. 41-62, 1996.

TILLMAN, H. N. Evaluating quality on the Net. 1997. Disponível em: http://www.tiac.net.users/. Acesso em: 20 out. 2015.

Data de submissão: 12 de agosto de 2019.

Data de aceitação: 26 de dezembro de 2019.

${ }^{1}$ BRASIL. CONSELHO DE GOVERNO COMITE EXECUTIVO DO GOVERNO ELETRÔNICO. Resolução
$\mathrm{n}^{\mathrm{o}} \quad 7, \quad 29 \quad$ de
http://www.planalto.gov.br/ccivil_03/Resolu\%C3\%A7\%C3\%A3o/2002/RES07-02web.htm/. Acesso em 15.05 .2018$.

2 CREMESP. Disponível em: https://www.cremesp.org.br/? Acesso em: 24 set. 2015. Acesso em: 05 de maio de 2018.

$\begin{array}{cccc}3 & \text { Disponível } & \text { em: } & \text { http://www.rfc-base.org }\end{array}$

